\title{
Globe
}

Revue internationale d'études québécoises

\section{Note critique. Identités et droits indigènes : six ouvrages récents sur les Autochtones}

\section{Louis-Jacques Dorais}

Volume 8, numéro 1, 2005

URI : https://id.erudit.org/iderudit/1000902ar

DOI : https://doi.org/10.7202/1000902ar

Aller au sommaire du numéro

Éditeur(s)

Globe, Revue internationale d'études québécoises

ISSN

1481-5869 (imprimé)

1923-8231 (numérique)

Découvrir la revue

Citer cette note

Dorais, L.-J. (2005). Note critique. Identités et droits indigènes : six ouvrages récents sur les Autochtones. Globe, 8(1), 221-236.

https://doi.org/10.7202/1000902ar d'utilisation que vous pouvez consulter en ligne.

https://apropos.erudit.org/fr/usagers/politique-dutilisation/ 


\section{Recensions}

\section{Note critique. Identités et droits indigènes : six ouvrages récents sur les Autochtones ${ }^{1}$}

Parmi les dizaines d'ouvrages en français sur les Autochtones du Québec et du Canada parus au cours des dernières années, nous allons examiner six titres que l'on peut relier, malgré la diversité apparente des sujets, à la problématique des droits territoriaux et de l'identité amérindienne et inuit dans le monde d'aujourd'hui.

Dans La gestion de l'étranger, l'ethnohistorien Claude Gélinas se penche sur l'histoire culturelle des Atikamekw de la Haute-Mauricie de 1760 à 1870. Il s'agit d'un travail extrêmement fouillé, où l'auteur examine tour à tour la vie traditionnelle (avant 1760) des Atikamekw, les principales caractéristiques de leur vie sociale et de leur culture au temps du commerce des fourrures (1760-1831), ainsi que les contacts qu'ils ont entretenus avec la Compagnie de la baie d'Hudson et les missionnaires catholiques à partir de 1831, contacts qui ont mené à leur sédentarisation progressive après 1850 .

1. Cet article recense les ouvrages suivants: Claude Gélinas, la gestion de l'étranger. Les Atikamekw et la présence eurocanadienne en Haute-Mauricie, 1760-1870, Québec, Septentrion, 2000 ; Nelson-Martin Dawson, Des Attikamègues aux Têtes-de-Boule. Mutation ethnique dans le Haut Mauricien sous le Régime français, Québec, Septentrion, 2003 ; Jean-Jacques SIMARD, La réduction. L'Autochtone inventé et les Amérindiens d'aujourd'bui, Québec, Septentrion, 2003; Tom Flanagan, Premières nations? Seconds regards [traduit de l'anglais par Pierre Desrosiers], Québec, Septentrion, 2002 ; Jean-Paul LACASSE, Les Innus et le territoire. Innu tipenitamun, Québec, Septentrion, coll. * Territoires ", 2004 et Frédéric LAUGRAND, Mourir et renaître. La réception du christianisme par les Inuit de l'Arctique de l'Est canadien (1890-1940), Québec, Presses de l'Université Laval, coll. “ Religions, cultures et sociétés „, 2002.

Karim Larose [éd.], - Recensions ", Globe. Revue internationale d'études québécoises, vol. $8, \mathrm{n}^{\circ} 1,2005$. 
L'étude de Gélinas est un modèle d'érudition et de clarté. Reprenant la quasi totalité des études et documents d'archives touchant les Atikamekw, elle offre un panorama complet et, à mon avis, définitif ou presque de l'histoire culturelle de ce peuple à une époque de contacts croissants avec les Euro-Canadiens. L'auteur conclut que depuis le xvil siècle, la Haute-Mauricie a vécu divers mouvements de population d'Algonquiens nomades qui entretenaient des contacts, parfois de longue distance, avec d'autres Algonquiens, des Iroquoiens et des Européens. Malgré ces contacts, qui ont toujours fait partie du mode de vie des Autochtones de la Haute-Mauricie, c'est le petit groupe de chasse plutôt que la * tribu * ou la bande régionale - qui a constitué l'unité sociale de base jusqu'en 1870 .

Selon l'auteur, l'interprétation des sources consultées montre que les Autochtones qui occupaient la Haute-Mauricie jusqu'au xvII siècle ne différaient en rien, sauf par des particularités mineures touchant la langue et l'adaptation à l'environnement, des autres Algonquiens établis à l'est et à l'ouest de leur territoire. Ce facteur d'homogénéité linguistique et culturelle, couplé au nomadisme, ne permet pas, selon Gélinas, de prendre position sur l'existence ou non d'une continuité ethnique entre les Atikamekw du début du Régime français et ceux qu'on a appelés - Têtes-de-Boule - à l'époque de la traite des fourrures (et qui sont redevenus des Atikamekw au tournant des années 1970). S'agit-il ou non du même peuple ? L'auteur semble croire - et j'estime qu'il a raison sur ce point - que la notion de continuité ou d'identité " ethnique * n'est pas pertinente dans le cas de populations nomades qui ne se distinguaient pas vraiment de leurs voisins algonquiens. Certains groupes de chasse ont pu occuper la Haute-Mauricie à certaines époques, pour migrer ailleurs après quelques années, voire quelques générations, et éventuellement y revenir - ou avoir des descendants qui y revenaient - plusieurs décennies plus tard. D'autres groupes, par contre, ont sans doute continué à occuper le territoire, quitte à en émigrer et peut-être à y immigrer de nouveau eux aussi à un autre moment de leur histoire.

Dans Des Attikamègues aux Têtes-de-Boule, l'historien Nelson-Martin Dawson se pose justement la question de la continuité de l'occupation autochtone dans la Haute-Mauricie. Il se demande si les "Attikamègues * rencontrés là-bas par le père Buteux en 1652 et les "Têtes-de-Boule . contactés près de deux cents ans plus tard (1837) par un autre ecclésiastique, l'abbé Dumoulin curé de Yamachiche, forment un seul et même 
peuple. Dawson cite diverses interprétations (dont celle de Gélinas qu'il interprète - à tort me semble-t-il, on vient de le voir - comme l'affirmation de la continuité ethnique des Autochtones de la région) appuyant la thèse de l'identité entre a Attikamègues - et " Têtes-de-Boule * ou allant à son encontre. Un examen attentif des textes et autres documents d'époque lui permet ensuite de se ranger contre cette thèse. D'après l'interprétation qu'il fait de ces documents, l'auteur conclut que les a Attikamègues " du xvir siècle, se sont éteints à la suite des guerres iroquoises et des épidémies. Selon Charlevoix, cette extinction aurait été complète, mais selon d'autres sources, un petit nombre d'Autochtones aurait survécu et aurait été absorbé par les migrants amérindiens qui seraient ensuite venus s'installer dans le territoire décimé de la Haute-Mauricie.

Ces nouveaux occupants, appelés par les Français "Sauvages des terres * puis "Têtes-de-Boule ", seraient originaires de la région du lac Supérieur. Selon Dawison, l'appellation "Tête-de-Boule " viendrait de "Tête-de-Bœuf " (par contamination de l'anglais "bull", "bœuf "). Il s'agirait d'une déformation du mot algonquien "ackipo ", lui-même une forme du terme "eskimau ", appliqué par les Algonquiens à tous les peuples étrangers (dont les Inuit). Les "Têtes-de-Boulle * seraient donc des "Esquimaux ".

On peut s'interroger sur les habiletés linguistiques de Dawson. Ses capacités d'historien sont sans doute meilleures et je lui accorde le bénéfice du doute quant à son examen des sources d'archives. Il m'est impossible par contre de juger de son évaluation finale de la situation, qui veut qu'en aucun cas les "Attikamègues * du xvil siècle puissent être identifiés aux "Têtes-de-Boule • du XXXe, redevenus des Atikamekw à la fin du $x x^{e}$ siècle. Il s'agirait de deux peuples tout à fait différents, même si l'auteur admet la possibilité que des survivants "attikamègues " aient pu s'intégrer aux migrants * têtes-de-boule "

Cette question ne constituerait qu'un épisode, somme toute assez mineur, de l'histoire des Autochtones du Québec si, comme Dawson le mentionne en début d'ouvrage, l'absence de continuité * ethnique * entre "Attikamègues * et " Têtes-de-Boule " ne remettait pas en cause, selon lui, le titre autochtone que les Atikamekw d'aujourd'hui croient détenir sur la Haute-Mauricie. L'auteur souligne en effet que selon les critères légaux actuels, un tel titre repose sur la continuité du peuplement. Y a-t-il continuité dans le cas qui nous occupe ? Est-elle démontrable (ou son 
contraire l'est-il) dans le cas de groupes de chasse nomades peu différenciés de leurs voisins, comme les définit Gélinas? Et comment tenir compte du fait que d'éventuels rescapés " attikamègues " aient pu, selon Dawson, s'intégrer aux immigrants " têtes-de-boule "? Leurs descendants auraient-ils des droits que les autres n'auraient pas? Si les Atikamekw d'aujourd'hui ne détiennent pas de titre sur la Haute-Mauricie, pourraientils en détenir un sur leur région d'origine quelle qu'elle soit ? Et si c'est le cas, devrait-on procéder à des échanges de populations comme entre l'Inde et le Pakistan en 1947 ? Ou à des expulsions comme dans la Palestine de 1948 ? La question reste ouverte et elle dépasse de beaucoup les finasseries légales et historiques des spécialistes.

L'ouvrage du sociologue Jean-Jacques Simard, La Réduction, tente précisément d'inscrire la question autochtone dans un cadre beaucoup plus vaste. Il s'agit d'un recueil d'articles et d'autres textes (23 au total) publiés, pour la plupart (quelques textes sont inédits), par l'auteur entre 1972 et 2002, soit au long d'une période de 30 ans. Le livre de Simard lui a valu un Prix littéraire du Gouverneur général en 2004.

L'idée maîtresse de l'auteur, qui a peu varié au cours des années et sur laquelle les textes du recueil constituent des variations, c'est que la notion d' Autochtone " ne devient significative que dans le miroir des " envahisseurs venus d'ailleurs " - lire les Européens qui ont peuplé le Nouveau Monde à compter du $\mathrm{xv}^{\mathrm{e}}$ siècle. L'Autochtone n'existe pas en soi. Il a été inventé par les anthropologues et autres idéologues de l'ethnicité, qui ont dû créer un "Autre ", le "Sauvage ", conçu comme l'envers du " Soi * civilisé, pour, à la fois, démontrer leur différence - et donc leur supériorité - et maintenir une distance entre les “ Blancs * et les populations locales. Cette différence supposée et la distance qu'elle engendrait ont d'abord servi à justifier la prise de possession des territoires amérindiens par les Européens, puis, beaucoup plus récemment, à la suite d'un retour du balancier, à défendre les pseudo-droits de ces Autochtones imaginaires.

L'ouvrage de Simard ne traite donc pas de l'ethnologie des autres (culture traditionnelle, langue, etc.), sujet que l'auteur semble juger sans grand intérêt ni véritable pertinence - autre qu'anecdotique - de nos jours. Il s'intéresse plutôt à la situation des Amérindiens dans l'environnement social où ils s'inscrivent depuis 1492, ainsi qu'à l'évolution récente et aux effets contemporains de l'interaction inégale entre popula- 


\section{RECENSIONS}

tions locales et migrants d'origine européenne, au Québec et au Canada. Pour les Amérindiens, cette interaction a principalement consisté en un exil physique de l'espace (l'enfermement dans les réserves) et un exil mental du temps (la négation d'une possible modernité), donc en une réduction, pour reprendre un terme créé par les jésuites du $\mathrm{XvI}^{\mathrm{e}}$ siècle. Cette réduction a entraîné une dépendance généralisée chez les "Autochtones ", auxquels on a à peu près totalement interdit toute possibilité de se prendre en main. "Autochtones - et "Blancs "doivent donc sortir ensemble de la réduction en reconnaissant la modernité des premiers et leur droit à ne pas être différents.

Comme je l'ai mentionné plus haut, les cinq parties du livre constituent des variations sur ce thème de l'Autochtone inventé, réduit territorialement et conceptuellement dans sa relation avec la société d'aujourd'hui. L'auteur s'intéresse tour à tour à l'invention du concept d' Autochtone", au rôle de l'acteur autochtone dans la société québécoise, à la situation particulière des Inuit, à l'expérience de la Baie-James, et à la nécessité de dire adieu à l'Autochtone imaginaire pour se rendre enfin compte qu'Indiens et Inuit vivent à la même époque et dans la même société que leurs concitoyens québécois. Pour Simard, il est faux d'affirmer, comme le font les discours académiques et politiques dominants (la Commission royale d'enquête sur les peuples autochtones, qui a siégé de 1991 à 1996, par exemple), qu'il existe des "Autochtones * ayant un mode de vie radicalement distinct de celui des "Blancs ", mode de vie qui n'aurait pas vraiment changé depuis les temps précolombiens et qui justifierait l'octroi de droits territoriaux et politiques spéciaux aux Amérindiens et aux Inuit.

Dans l'ensemble, les propositions de Simard sont justes et remettent certaines pendules à l'heure. Il est inexact et non constructif, par exemple, de considérer les "Autochtones " comme enfermés dans une a culture traditionnelle , figée dans le temps, qui en ferait des êtres à part possédant une nature différente de celle du reste de la population. Le Québec et le Canada modernes ne peuvent se construire que dans l'égalité - non seulement sociale, mais aussi symbolique - entre tous leurs citoyens, quelle que soit l'origine de ceux-ci et leur date d'arrivée en Amérique du Nord.

Par contre, les propos de l'auteur ont parfois aussi leurs faiblesses. Simard met le doigt sur des visions simplistes et des idées reçues (le 
"traditionalisme autochtone "), mais sans toujours se rendre compte qu'il existe d'autres discours, provenant même souvent d'anthropologues, qui insistent justement sur la modernité des Amérindiens et des Inuit, sur leurs pratiques d'individus pleinement modernes et actifs dans le monde contemporain. Son analyse de la réduction, aussi intéressante qu'elle soit, l'empêche peut-être de voir que la dépendance autochtone est loin d'être absolue, les populations locales ayant depuis toujours su manipuler et manœuvrer à leur insu les commerçants, missionnaires, administrateurs et chercheurs installés chez elles. Comme certains textes de Simard ont été écrits à des époques éloignées les unes des autres, il n'est pas étonnant qu'ils se contredisent parfois en partie. Si l'auteur estime, par exemple, que le mode de vie des Indiens et des Inuit n'est maintenant plus distinct de celui des * Blancs " (partie V de l'ouvrage), il affirme ailleurs (partie II) que les a Autochtones "ont droit à l'autosuffisance économique, à l'autonomie politique et à l'autodéfinition de leur horizon culturel. Sur quoi un tel droit serait-il fondé s'il n'y a aucune différence de mode de vie entre Amérindiens et Européens?

En fait, Simard semble sous-estimer l'importance de l'identité. Celleci repose souvent sur des éléments subtils, difficiles à repérer (on en aura des exemples plus loin lorsque nous examinerons les ouvrages de Lacasse et de Laugrand), qui peuvent donner l'impression que les "autres " sont semblables à nous, mais qui font que ces autres, eux, se croient différents. Les modernités amérindiennes et inuit ne sont peutêtre pas exactement semblables à celles des francophones, des anglophones ou des autres * allochtones * du Québec. Nous ne sommes peutêtre pas " emportés par les mêmes tendances, modes, institutions, aspirations, etc. ${ }^{2}$ " de la même façon les uns que les autres. C'est sur ces différences que se fondent les identités. S'il y a au Québec, au Canada ou ailleurs des individus ou des groupes qui se croient * autochtones ", est-ce simplement parce que des anthropologues, des bureaucrates ou des élites amérindiennes avides de pouvoir les ont persuadés de cela? Ces soi-disant * Autochtones " sont-ils des imbéciles ou des ignorants qui ne savent pas analyser leur situation et définir leur bien commun aussi habilement que ne le font les sociologues? Les droits qu'ils réclament ne serviront-ils qu'à perpétuer leur dépendance et une forme proprement

2. Jean-Jacques SIMARD, La réduction. L'Autochtone inventé et les Amérindiens d'aujourd'bui, Québec, Septentrion, 2003, p. 394. 
canadienne d'apartheid, ou leur permettront-ils au contraire de proclamer et de vivre une modernité autre?

La réponse à ces questions n'est pas évidente. On peut admettre, avec des anthropologues (eh oui!) comme Adam Kuper ${ }^{3}$, que le mouvement actuel en faveur des droits " autochtones " repose sur des notions dépassées. C'est une simple réaction aux revendications écologistes et antimondialistes qui crée des identités artificielles. On peut aussi supposer, avec Jonathan Friedman ${ }^{4}$, que les "autres * sont des personnes réelles luttant pour contrôler leurs conditions d'existence, dans le cadre d'une identité sociale (qualifiée d' indigène ") d'un type particulier. Cette identité se constitue autour de continuités culturelles liées à une expérience d'enracinement, que reflètent mal nos catégories d'analyse habituelles. D'où sa légitimité.

Les questions soulevées par Simard sont reprises de façon percutante dans le livre du politologue Tom Flanagan, l'un des principaux maîtres à penser de la nouvelle droite canadienne. Premières nations? Seconds regards a d'abord été publié en anglais en l'an 2000, et sa publication a soulevé bien des controverses. C'est pourquoi l'éditeur francophone de l'ouvrage a cru bon de faire précéder celui-ci d'une préface de Guy Laforest, politologue lui aussi et ci-devant président de l'Action démocratique du Québec, et de le faire suivre des commentaires de quatre "éminents chercheurs québécois ${ }^{5}$ : l'économiste Jean-Luc Migué, le juriste Ghislain Otis, le sociologue JeanJacques Simard (le texte de son commentaire a d'ailleurs été repris dans La réduction) et le philosophe politique Charles Taylor.

Un peu comme Simard, mais de façon beaucoup plus directe, Flanagan se pose d'entrée de jeu en contradiction avec le discours dominant sur la condition autochtone. À son avis, si le type de relations avec les Amérindiens et les Inuit préconisé par la Commission royale sur les peuples autochtones et les décisions récentes des tribunaux se développe, "le Canada sera redéfini comme un État multinational

3. Adam KuPER, - The Return of the Native *, Current Anthropology, vol. 44, $\mathrm{n}^{\circ} 3$, 2003.

4. Jonathan Friedman, - Indigenous struggles and the discreet charm of the bourgeoisie *, The Australian Journal of Antbropology, vol. 10, n 1, 1999.

5. Quatrième de couverture. 
comprenant un archipel de nations autochtones qui seront propriétaires d'un tiers du territoire canadien, exemptes d'impôts fédéraux et provinciaux, économiquement soutenues par des paiements de transfert provenant des contribuables, autorisées à ne plus se soumettre aux lois fédérales et provinciales, et libres d'entretenir des relations diplomatiques "de nation à nation" avec ce qui restera du Canada ${ }^{6}$. Pour échapper à cette situation apocalyptique, il faut renverser "l'orthodoxie autochtone " qui repose sur huit propositions (faisant chacune l'objet d'un chapitre) dont l'ouvrage de Flanagan cherche à démontrer la fausseté :

1) Les Autochtones détiennent des droits particuliers parce qu'ils ont été les premiers à occuper le territoire - mais nous sommes tous des immigrants, tant Autochtones que Nord-Américains d'origine européenne ou autre, et distinguer entre les droits des premiers et des demiers arrivés frise le racisme.

2) Les cultures autochtones sont au même niveau que celle des colonisateurs européens - faux ! La civilisation européenne était en avance de plusieurs millénaires sur les cultures autochtones. La colonisation de l'Amérique du Nord était donc inévitable et justifiable.

3) Les peuples autochtones étaient souverains - encore faux! La souveraineté est un attribut relevant des États. Or, les Autochtones n'avaient et n'ont toujours pas d'États. Ils n'ont donc pas droit à la souveraineté.

4) Les Autochtones constituent des nations au sens culturel et politique - il ne saurait exister au Canada qu'une seule communauté politique suprême, donc une seule nation canadienne.

5) Le droit inhérent à l'autonomie gouvernementale peut s'exercer sur le territoire des réserves - mais l'exercice d'une gouvernance autochtone sur des territoires restreints engendre presque toujours des querelles familiales inutiles et destructrices.

6) Les droits de propriété autochtones devraient être constitutionnellement reconnus, plutôt qu'éteints - mais l'exercice de ces droits tels que définis par les tribunaux est impossible dans les économies modernes.

6. Tom Flanagan, Premières nations? Seconds regards, Québec, Septentrion, 2002, p. 15. 
7) Les traités signés avant l'époque contemporaine ont un autre sens que ce que leur libellé laisse entendre - en réalité, ces traités signifient bien ce qu'ils disent. Leur redéfinition serait coûteuse et dangereuse pour l'économie des provinces.

8) Les Autochtones vivant et travaillant sur leur territoire deviendront prospères - faux! La prospérité est impossible à atteindre si on ne s'intègre pas à l'économie générale, ce qui suppose la mobilité de la main-d'œuvre.

L'ouvrage de Flanagan est extrêmement bien documenté et son raisonnement est fondé sur des prémisses rigoureuses. Chacun des arguments mis de l'avant par l'auteur pour réfuter les huit propositions qu'il juge fausses est plausible et se base sur des faits bien démontrés, exception faite peut-être de quelques erreurs mineures d'interprétation. Ce texte donne donc à réfléchir.

Pour Flanagan, le droit de conquête par une population civilisée la civilisation étant ici définie par la présence simultanée, chez un peuple donné, de l'agriculture, de l'urbanisation, de la division du travail, du progrès intellectuel, d'une technologie avancée et d'un appareil d'État et techniquement supérieure est normal et souhaitable. Les Européens constituent la quatrième " tribu " (après la majorité des Amérindiens, les Dènè, puis les Inuit) à avoir occupé l'Amérique. Ils en ont bousculé les occupants tout comme leurs prédécesseurs l'avaient fait avant eux. Puisque leur civilisation était supérieure aux cultures locales, il est normal qu'elle les ait peu à peu remplacées. Cela va donc à l'encontre du progrès que de tenter de " réduire o les Autochtones - pour reprendre l'expression de Simard - en les enfermant dans de pseudo-droits qui les mettent à l'écart du développement national du Canada et qui risquent à la longue de nuire à ce développement.

La problématique de Flanagan, qui est celle de la droite conservatrice néo-libérale (l'auteur est l'un des conseillers les plus écoutés de Stephen Harper, le chef du Parti conservateur), est nettement inspirée de l'évolutionnisme darwinien. Il est normal - et c'est même voulu par Dieu pour certains - que le plus fort élimine le plus faible, que le civilisé remplace le sauvage. Cette vérité inéluctable justifie le colonialisme et plus particulièrement, dans le cas présent, l'occupation et le développement des Amériques par des Européens en tout supérieurs aux populations autochtones. Ces dernières ont donc intérêt, pour leur plus 
grand bien, à poursuivre leur assimilation, d'ailleurs déjà très avancée, aux systèmes économique, politique et culturel mis en place par les colonisateurs. Flanagan conclut son ouvrage en soulignant que l'" orthodoxie autochtone " est cohérente, mais que sa logique interne n'a pas d'utilité politique et économique concrète. Il faut donc la mettre de côté pour "civiliser " les Premières Nations, en créant chez elles les conditions d'émergence d'une société civile à l'image de la nôtre.

Que penser d'une telle façón d'envisager les choses? Je l'ai mentionné plus haut, les propos de l'auteur sont d'une logique sans faille et reposent sur des faits et des arguments solides et extrêmement bien documentés. Mais sont-ils acceptables pour autant?

Deux des quatre auteurs appelés à commenter l'ouvrage de Flanagan se montrent d'accord avec ses positions, les deux autres y apportent de plus ou moins gros bémols. Pour Jean-Luc Migué, la société doit reconnaître que "l'accès à l'éducation et à la richesse ne peut que supplanter les valeurs des peuples aborigènes. L'intégration à l'économie universelle et l'autonomie culturelle des Indiens sont incompatibles ${ }^{7}$. Il est normal que le capitalisme et la démocratie libérale, qui seuls peuvent assurer la croissance, remplacent les cultures autochtones. Il faut donc abandonner au plus vite toute politique spécifique aux Premières Nations.

Pour Jean-Jacques Simard, Flanagan démontre avec rigueur le fondement futile des politiques actuelles envers les Autochtones. Les propos de son ouvrage sont donc acceptables. Simard, qui possède une fibre sociale faisant totalement défaut à Migué, regrette toutefois que le libéralisme extrême de Flanagan amène celui-ci à rejeter toute forme d'aide gouvernementale visant à corriger les inégalités structurelles qui défavorisent certaines minorités, y compris une bonne partie des Amérindiens et des Inuit.

Ghislain Otis, lui, n'est pas convaincu par la remise en cause du fondement même des droits autochtones. Le livre de Flanagan aurait cependant le mérite de nous amener à nous demander si le droit hérité de l'époque coloniale est vraiment adapté au besoin de développement

7. Jean-Luc Migú dans Tom Flanagan, Premières nations? Seconds regards, Québec, Septentrion, 2002, p. 209. 
et d'autosuffisance des Autochtones d'aujourd'hui. Quant à Charles Taylor, s'il félicite Flanagan de contribuer - quoique de façon brutale à assainir le débat, il insiste aussi sur l'importance de reconnaître l'Autre tel qu'il est et tel qu'il veut être. On ne doit pas imposer aux Autochtones la vision que nous avons d'eux - Taylor rejoint ici Simard -, vision déformée que les Autochtones adoptent souvent eux-mêmes. Il faut plutôt reconnaître franchement les ressemblances et les différences qui rapprochent et séparent anciens et nouveaux occupants du territoire nordaméricain. Il est donc nécessaire de comprendre les identités autochtones, au lieu de les interpréter ou de les juger, car l'identité a préséance sur la force.

En somme, l'acceptation ou le rejet des arguments de Flanagan, tout logiques et bien informés qu'ils soient, dépend du paradigme philosophique et moral à l'intérieur duquel on se situe. Si on estime que la croissance à tout prix et la liberté individuelle absolue constituent des idéaux incontournables, alors Flanagan a raison. Par contre, si on croit qu'il existe d'autres valeurs que la rationalité économique, et si on pense - à tort ou à raison - que l'identité la plus profonde peut reposer sur des représentations autres que celles de l'Occident moderne, on est peut-être en droit de rejeter les arguments de Premières nations? Seconds regards.

C'est une telle attitude de rejet qu'adopte implicitement le juriste (directeur du programme de préparation aux études juridiques pour Autochtones à l'Université d'Ottawa) Jean-Paul Lacasse, dans Les Innus et le territoire. Sans jamais citer Flanagan, cet auteur considère que malgré l'absence d'État et de notion de propriété privée, les Innus (autrefois appelés Montagnais) ont toujours possédé un ordre légal et un système de gouvernance - fondés sur des pratiques coutumières plutôt que sur des institutions formelles - de nature proprement politique qui subsistent encore aujourd'hui. Le titre aborigène (droit autochtone fondamental) au Nitassinan (territoire où vivent les Innus) repose donc sur une identité culturelle faite de représentations mentales et de relations sociétales différentes de celles qui fondent le droit eurocanadien, et non sur un mode de vie qui, à toutes fins pratiques, ressemble maintenant beaucoup au nôtre.

Le grand mérite du livre de Lacasse est de donner la parole aux Innus (le texte est parsemé de citations intégrales d'interlocuteurs autochtones) et d'analyser les concepts qui, dans leur langue, expriment 
la nature et le fonctionnement de l'ordre cosmologique et social (contrairement à ce qui se passe chez nous, celui-ci n'est pas perçu de façon dichotomique). L'ouvrage se veut une contribution - extrêmement bien documentée - à la vision innue de ce que nous appelons le droit et à l'application des principes de droit innu au Nitassinan.

L'univers innu est perçu comme fondé sur la notion de gardiennage, plutôt que de propriété, de la terre. Ce qui importe avant tout, c'est la bonne gestion des ressources au profit de la famille et de la communauté. Aux yeux des Innus, le fait d'occuper le territoire depuis toujours est donc aussi valable qu'un titre de propriété. Il n'y a jamais eu d'État innu organisé, mais la notion de juridiction (tipenitamun) existe. Celleci inclut les concepts de souveraineté, de valeurs et d'ordre social, sur lesquels reposent des normes de conduite précises et un devoir de gestion bien délimité.

L'occupation du Nitassinan par les Européens était peut-être justifiable selon le droit international de l'époque, mais les Innus l'ont ressentie comme une intrusion allant à l'encontre de leur ordre juridico-politique propre. Ils ont conservé la forte impression d'avoir été les premiers à occuper leur territoire et de ne l'avoir jamais cédé à quiconque. Dès le $\mathrm{XIX}^{\mathrm{e}}$ siècle, des Innus ont envoyé des pétitions au gouvernement pour protester contre l'implantation européenne. La création de réserves a pu servir de rempart contre l'assimilation, mais elle n'a pas effacé leur très vif sentiment d'avoir été dépossédés. Comme l'ordre juridique autochtone n'était pas reconnu par les Européens, ceux-ci se sont implantés en force au Nitassinan, ce qui a entraîné des transformations brutales de la société innue et la marginalisation de la population autochtone. Cette marginalisation n'a toutefois pas empêché la persistance d'une forte conscience territoriale.

Deux titres de propriété légale s'affrontent donc au Nitassinan. Celui de la Couronne, fondé sur le droit colonial amendé par la constitution canadienne de 1982 telle qu'interprétée par la Cour suprême, et celui des Innus. Ce dernier repose sur l'occupation première (même nomade) du territoire, sur la toponymie en langue autochtone et sur l'existence d'un ordre juridique indigène de gestion de la terre. Le titre innu est bien démontré et le droit à l'autonomie gouvernementale qui en découle constitue lui aussi un droit ancestral inhérent. 
Lacasse conclut son ouvrage en affirmant qu'on doit permettre aux Innus de conserver leur Innu tipenitamun (juridiction autochtone), parce que l'histoire montre que les tentatives d'intégration totale à la société québécoise ont mené à l'échec. Malgré l'acculturation marquée, les valeurs autochtones ont subsisté chez les jeunes. Il faut reconnaître la légitimité du fait que les Innus jugent toujours leurs valeurs et leurs règles de conduite comme inséparables du territoire et de l'autonomie de gestion. On doit donc écouter les habitants du Nitassinan, qui veulent développer un projet de modernité endogène ouvert aux échanges mutuels.

L'étude de Lacasse me semble démontrer - a contrario de ce qu'avancent Flanagan et, avec plus de nuances, Simard - qu'au-delà des grandes oppositions universel/local ou modernité/tradition, il existe des pratiques, des modes d'interrelation et des représentations qui, tout en se démarquant des nôtres, n'en jouent pas moins un rôle économique, politique et identitaire équivalent. Ces pratiques, modes et représentations - souvent masqués sous une apparence de conformité quasi totale au mode de vie nord-américain - ne doivent-ils pas être respectés? La revendication par les Autochtones du droit à une modernité différente ne justifie-t-elle pas la légitimité d'institutions spécifiques protégeant cette façon différente d'être moderne ? Je ne peux juger de la validité des analyses de Lacasse sur la société innue, mais si elles reflètent vraiment la pensée populaire - et non celle des seules élites, comme Simard et Flanagan le croient -, le titre autochtone sur le Nitassinan et sur sa gestion me semble totalement justifié.

Dernier ouvrage à être examiné ici, le livre de l'anthropologue Frédéric Laugrand, Mourir et renaitre, se différencie des autres à certains égards. C'est le seul à porter sur les Inuit. Le seul aussi à ne pas avoir été publié par les éditions du Septentrion. Il s'agit d'une étude en profondeur du processus de christianisation des Inuit de l'Arctique de l'Est canadien (Nunavut et nord du Québec). Comme les ouvrages précédents, celui-ci se caractérise par une recherche fouillée en bibliothèque et en archives, recherche que Laugrand a combinée - trait absent des autres travaux recensés ici - à une étude de terrain au cours de laquelle il a rencontré et interrogé directement les descendants (et même quelques témoins encore vivants) et les héritiers spirituels des protagonistes inuit et missionnaires du processus de conversion. 


\section{REVUE INTERNATIONALE D'ÉTUDES QUÉBÉCOISES}

L'auteur essaie de comprendre comment les Inuit en sont venus à adhérer au christianisme en seulement un demi-siècle. Son étude se veut à la fois descriptive et structurale. Elle examine une forme spécifique d'interaction où il existe déjà des points d'ancrage entre Soi et l'Autre. Même si des idées chrétiennes avaient couru dans certaines régions de l'Arctique avant le $\mathrm{xIX}^{\mathrm{e}}$ siècle, par contact direct ou indirect, ce n'est qu'à partir de la seconde moitié du siècle que la circulation de ces idées devint plus fluide et que le prosélytisme se développa. Au cours des premières décennies du $\mathrm{Xx}^{\mathrm{e}}$ siècle, la disparition de pratiques chamaniques importantes à la suite de l'émergence d'un certain modernisme des idées et des pratiques dû au contact avec les Euro-Canadiens, engendra une situation favorable pour les missionnaires, qui arrivèrent ainsi à un moment opportun. Comme les évangélisateurs - anglicans et catholiques - étaient peu nombreux et ne pouvaient se retrouver partout à la fois, les prosélytes inuit eurent un rôle important à jouer dans la diffusion du christianisme. C'est pourquoi on peut distinguer entre deux grands contextes de christianisation : les zones de contacts directs et les zones de contacts indirects.

Dans les premières, où les missionnaires prêchaient la bonne parole en personne, la conversion fut plus ardue en raison de l'hostilité des chamans (angakkuit) qui toléraient mal l'arrivée de ces étrangers venus leur faire concurrence. Ce n'est qu'après plusieurs années que les angakkuit, finalement convaincus de la supériorité des nouveaux esprits, acceptèrent de se convertir, précédés ou suivis des membres de leur groupe. Les motifs réels de conversion étaient multiples, mais on peut distinguer trois phases générales: 1) prise de conscience de la situation nouvelle; 2) décision d'adopter le christianisme ; 3) incorporation par le baptême. Dans tous les cas, la nouvelle structure religieuse devait répondre aux besoins des convertis et prendre des formes signifiantes pour eux.

La diffusion du christianisme se fit plus facilement dans les zones de contacts indirects, à cause de facteurs structurels et conjoncturels souvent redevables à l'action des prosélytes inuit qui y pénétrèrent avant les missionnaires. Ces prosélytes, convertis dans le sud du territoire inuit à la suite de leurs contacts avec les Euro-Canadiens, introduisirent au nord une lecture - que Laugrand qualifie de a frénétique " - de la Bible, qui coïncida souvent avec la disparition, mentionnée plus haut, de certaines pratiques chamaniques. Tout ceci prépara le terrain pour les missions, qui profitèrent de ce contexte favorable pour s'étendre à l'ensemble du 
territoire. La concurrence entre anglicans et catholiques atteignit son paroxysme à la fin des années 1920 et au cours des années 1930. Le processus de conversion s'accéléra avec la multiplication des contacts, mais les rivalités confessionnelles le ralentirent quelque peu.

Laugrand conclut de tout cela qu'on peut dégager quatre grands facteurs expliquant la réception relativement rapide du christianisme par les Inuit de l'Arctique de l'Est canadien : 1) Les Inuit montraient de la reconnaissance envers la faculté d'adaptation des missionnaires, qui apprenaient leur langue et vivaient parmi eux. 2) Ils interprétaient le christianisme selon les paramètres de leur propre culture; il y eut donc des déplacements culturels, mais pas de remplacement d'une culture religieuse par une autre. 3) L'apparition, dans les zones de contacts indirects, de mouvements parousiques autochtones prêchant le retour prochain du Christ et la fin du monde actuel, démontre l'existence d'un christianisme culturellement bricolé. 4) Les chamans trouvaient du sens dans la nouvelle religion, parce qu'elle ne remettait pas en cause leur responsabilité d'innover constamment en expliquant les changements sociaux et culturels vécus par les Inuit. L'institution chamanique, axée sur l'innovation, survécut ainsi à la disparition des chamans.

Cette institution chamanique perdure encore aujourd'hui à bien des égards. Les Inuit se sont donc appropriés le christianisme; il ne leur a pas été imposé. L'anglicanisme, le catholicisme et, devrait-on ajouter, le pentecôtisme - beaucoup plus récent -, aujourd'hui pratiqués dans l'Arctique canadien, diffèrent énormément de leurs modèles d'origine, même si en surface, les différences ne sont pas toujours perceptibles. L'étude de Laugrand montre ainsi que dans le domaine religieux, l'expérience cognitive des Inuit contemporains diverge sensiblement de celle des Euro-Canadiens.

On peut se demander encore une fois si de telles divergences de perception, qui ont leur pendant - on l'a vu avec Lacasse - dans le domaine juridico-politique et, sans doute, dans beaucoup d'autres champs (la conscience historique par exemple; voir à ce sujet l'ouvrage de Gélinas), ne fondent pas des identités autochtones profondément différentes des nôtres. En poussant la réflexion plus loin, on peut aussi s'interroger sur un lien possible entre identité et droits territoriaux. Malgré ce qu'en pensent Flanagan, Simard et Dawson, le désir d'affirmation et de développement d'identités amérindiennes et inuit modernes, 
mais différentes (sans être toutefois mythifiées) de celles de la population majoritaire, ne justifie-t-il pas l'exercice de droits particuliers?

La question reste ouverte, bien sûr. Les ouvrages que nous avons examinés ici, qu'ils relèvent surtout de l'érudition (Gélinas, Dawson et Laugrand), de la réflexion (Simard et Flanagan) ou d'un heureux mélange des deux (Lacasse), apportent cependant, chacun à leur façon, un éclairage utile et intéressant sur cette interrogation majeure du tournant du troisième millénaire.

Louis-Jacques Dorais Université Laval

\section{Geneviève Mativat}

L'Amérindien dans la lorgnette des juges.

Le miroir déformant de la justice.

Montréal, Recherches amérindiennes au Québec, 2003.

Au Canada, le droit autochtone s'inscrit dans la tradition juridique britannique de la common law, du fait qu'elle relève essentiellement de lois et de compétences fédérales. Dans cette approche, le législateur et les textes de loi demeurent des recours exceptionnels, pour lesquels s'impose une interprétation restrictive. Le droit se construit principalement en ayant recours aux juges et à leurs décisions, la jurisprudence de la Cour suprême demeurant l'ultime autorité. Son pouvoir est énorme sur le sens et la portée des contenus juridiques. Or, en vertu des théories constructivistes du droit, ses décisions s'inscrivent dans un imaginaire forgé par le contexte social, politique et culturel. Pour ces raisons, il s'imposait donc d'étudier la position de la Cour suprême dans une approche anthropologique et, plus encore, de déconstruire ses décisions afin de comprendre quels regards les plus hauts juges du pays portent sur les premiers peuples. Cet ouvrage trace ainsi un nécessaire mouvement en ce sens. Le fait que les Autochtones eux-mêmes semblent nourrir beaucoup d'espoirs à l'égard des tribunaux, tout en connaissant très mal leurs valeurs et leur imaginaire (p. 7), justifie d'autant plus cette démarche. 